\title{
Evaluation of the female pelvic floor in pelvic organ prolapse using 3.0-Tesla diffusion tensor imaging and fibre tractography
}

\author{
F. M. Zijta • M. M. E. Lakeman • M. Froeling • \\ M. P. van der Paardt • C. S. V. Borstlap • S. Bipat • \\ A. D. Montauban van Swijndregt • G. J. Strijkers • \\ J. P. Roovers • A. J. Nederveen · J. Stoker
}

Received: 15 January 2012 /Revised: 19 April 2012 / Accepted: 11 May 2012 /Published online: 14 July 2012

(C) The Author(s) 2012. This article is published with open access at Springerlink.com

\begin{abstract}
Objectives To prospectively explore the clinical application of diffusion tensor imaging (DTI) and fibre tractography in evaluating the pelvic floor.

Methods Ten patients with pelvic organ prolapse, ten with pelvic floor symptoms and ten asymptomatic women were included. A two-dimensional (2D) spin-echo (SE) echoplanar imaging (EPI) sequence of the pelvic floor was acquired. Offline fibre tractography and morphological analysis of pelvic magnetic resonance imaging (MRI) were performed. Inter-rater agreement for quality assessment of
\end{abstract}

F. M. Zijta $\cdot$ M. Froeling $\cdot$ M. P. van der Paardt $\cdot$ C. S. V. Borstlap $\cdot$

S. Bipat · A. J. Nederveen $\cdot$ J. Stoker

Department of Radiology, Academic Medical Centre, University

of Amsterdam,

Amsterdam, The Netherlands

F. M. Zijta $\cdot$ A. D. Montauban van Swijndregt

Amsterdam and Department of Radiology, Onze Lieve Vrouwe

Gasthuis,

Amsterdam, The Netherlands

M. M. E. Lakeman · J. P. Roovers

Amsterdam and Department of Gynaecology, Academic Medical Centre, University of Amsterdam the Netherlands and Biomedical NMR,

Amsterdam, The Netherlands

M. Froeling $\cdot$ G. J. Strijkers

Department of Biomedical Engineering, Eindhoven University of Technology,

Eindhoven, The Netherlands

\section{F. M. Zijta $(\bowtie)$}

Department of Radiology, Academic Medical Center,

Meibergdreef 9,

1105 Amsterdam, AZ, The Netherlands

e-mail: f.m.zijta@amc.uva.nl fibre tracking results was evaluated using weighted kappa $(\kappa)$. From agreed tracking results, eigen values $(\lambda 1$, $\lambda 2, \lambda 3$ ), mean diffusivity (MD) and fractional anisotropy (FA) were calculated. MD and FA values were compared using ANOVA. Inter-rater reliability of DTI parameters was interpreted using the intra-class correlation coefficient (ICC).

Results Substantial inter-rater agreement was found $(\mathrm{K}=$ 0.71 [95\% CI 0.63-0.78]). Four anatomical structures were reliably identified. Substantial inter-rater agreement was found for MD and FA (ICC 0.60-0.91). No significant differences between groups were observed for anal sphincter, perineal body and puboperineal muscle. A significant difference in FA was found for internal obturator muscle between the prolapse group and the asymptomatic group $(0.27 \pm 0.05$ vs $0.22 \pm 0.03 ; P=$ 0.015).

Conclusion DTI with fibre tractography permits identification of part of the clinically relevant pelvic structures. Overall, no significant differences in DTI parameters were found between groups.

Key Points

- Diffusion tensor MRI offers new insights into female pelvic floor problems.

- DTI allows 3D visualisation and quantification of female pelvic floor anatomy.

- DTI parameters from pelvic floor structures can be reliably determined.

- No significant differences in DTI parameters between groups with/without prolapse.

Keywords Magnetic resonance imaging (MRI) · Diffusion tensor imaging (DTI) · Pelvic floor disorders · Fibre tractography $\cdot$ Pelvic floor 


\section{Introduction}

Pelvic floor dysfunction entails a variety of conditions, including pelvic organ prolapse (POP), faecal and urinary incontinence. Certain basic risk factors have been considered, which among others include complicated vaginal delivery, normal ageing and obesity $[1,2]$. Although its precise pathophysiology is currently only partly understood, defects in the levator ani muscle are known to be highly associated with the presence of pelvic floor prolapse [3]. Therefore, visualisation of the pelvic floor musculature, identification of muscle injuries and, in particular, evaluation of muscle structure and function would complement generally used clinical tools, and might reveal aetiological factors for pelvic organ prolapse.

Both static and dynamic magnetic resonance imaging (MRI) enable a comprehensive interpretation of the complex pelvic floor anatomy and are increasingly proposed as a complementary diagnostic tool in pelvic floor dysfunction [4-7]. In the search for an improved three-dimensional (3D) understanding of anatomical relationships in the pelvic floor and changes in muscle integrity, which might correlate with pelvic floor dysfunction, recently the use of diffusion tensor imaging (DTI) with fibre tractography was proposed for the visualisation of the normal female pelvic floor [8].

Recent studies have reported DTI as a useful method for detecting alterations in tissue organisation of injured striated skeletal muscles compared with normal muscles [9, 10], which can be expressed in basic DTI parameters (i.e. eigen values, mean diffusivity [MD] and fractional anisotropy [FA]). The enhanced 3D visualisation with DTI and fibre tractography might have the potential to both visualise and quantify abnormal pelvic floor support in patients with prolapse, but has not been studied yet.

The purpose of this study was to examine the clinical application of DTI and fibre tractography of the pelvic floor support, by prospectively evaluating and comparing the fibre tract outcomes and basic DTI parameters of women with pelvic organ prolapse with those of women with pelvic floor symptoms but without pelvic organ prolapse, and with those of asymptomatic nulliparous women. We also intended to investigate the degree of inter-rater reliability.

\section{Materials and methods}

\section{Subjects}

For this prospective cross-sectional study, institutional review board approval was obtained and all participants gave written informed consent. Three different groups of subjects were included; in total 30 female subjects were enrolled in this study. Based on a published feasibility study [8], we anticipated the detection of (micro) structural differences between the symptomatic and asymptomatic pelvic support even in a small sample size.

The first group consisted of women with pelvic floor symptoms and at least a stage 2 pelvic organ prolapse as staged with Pelvic Organ Prolapse Quantification (POPQ) staging criteria, according to the recommendations of the International Continence Society (ICS) [11]. The second group, which was age-matched with the prolapse group, consisted of women who had pelvic floor symptoms but no clinically relevant pelvic organ prolapse (maximum stage 1). The third group consisted of nulliparous women, with no pelvic floor symptoms. All women were recruited in an outpatient clinic of a tertiary referral centre and underwent a clinical examination during which the presence of a prolapse was assessed and staged [11]. Pelvic symptoms comprised prolapse symptoms, defecation and/or micturition symptoms and were measured in all subjects using a disease-specific symptom questionnaire, which was based on the Urogenital Distress Inventory (UDI), Incontinence Impact Questionnaires (IIQ) and Defecation Distress Inventory (DDI) items $[12,13]$. Exclusion criteria for all groups included previous pelvic floor surgery and general contraindications to undergo MRI (e.g. pacemakers, claustrophobia and pregnancy).

\section{Image acquisition}

Each woman underwent MRI in the supine position at rest with the legs parallel, slightly flexed, using a 3.0-T MRI scanner (Intera, Philips Healthcare, Best, The Netherlands) with a 16-channel phased-array surface coil (SENSE-XLTorso, Philips Healthcare) for signal reception. No intravenous contrast medium was administered. Subjects were asked to empty the bladder $1 \mathrm{~h}$ before the examination. MRI comprised the acquisition of a multishot turbo spin echo (TSE) T1-weighted sequence (TR/TE, 600/10 ms; field-of-view [FOV], $200 \times 200 \mathrm{~mm}^{2}$; slice thickness, $5 \mathrm{~mm}$; slices, 20; echo train length, 7), multishot TSE T2weighted sequence $(\mathrm{TR} / \mathrm{TE}, 3,021 / 80 \mathrm{~ms}$; FOV, 300 $\times 300$ $\mathrm{mm}^{2}$; slice thickness, $4 \mathrm{~mm}$; slices, 31; slice gap, $0.4 \mathrm{~mm}$; echo train length, 16) in axial, coronal and sagittal planes for anatomical reference and dual-echo gradient echo imaging to derive a B0-field heterogeneity map (TR/TE1/TE2,12/ 4.6/9.6 ms; FOV, $200 \times 200 \mathrm{~mm}^{2}$; acquisition matrix, $80 \times$ 80; pixel size, $2.5 \times 2.5 \mathrm{~mm}^{2}$; slice thickness, $5 \mathrm{~mm}$; number of signal averages [NSA],2). Axial two-dimensional (2D) diffusion weighted images were acquired with a diffusion weighted spin-echo echo-planar imaging (SE-EPI) pulse sequence using the following MRI parameters: TR/TE, $3,750 / 40 \mathrm{~ms}$; FOV, $200 \times 200 \mathrm{~mm}^{2}$; acquisition matrix, $80 \times 80$; pixel size, $2.5 \times 2.5 \mathrm{~mm}^{2}$; slice thickness, $5 \mathrm{~mm}$; slices, 20; 32 diffusion weighted directions; NSA, 2; $b=$ 
$400 \mathrm{~s} / \mathrm{mm}^{2}$; spectral adiabatic inversion recovery [SPAIR] for fat suppression). The total acquisition time for the DTI sequence was less than $4 \mathrm{~min}$. The axial DTI sequence was non-angularly positioned and care was taken to place the anal sphincter complex and coccygeal bone within the FOV.

Post-processing and visualisation

DTI data were processed using a custom-built toolbox in Mathematica 8.0 [14]. Initially the data were filtered using a Rician noise suppression algorithm [15], after which the diffusion-weighted data were registered to the nonweighted images using an affine transformation and corresponding b-matrix rotation [16]. Subsequently the diffusion tensor was calculated and corrected for field heterogeneity-induced deformations. The per-voxel absolute vector direction was colour coded according to standardised FA map colour coding: red indicating the left-right direction, blue the superior-inferior direction and green the antero-posterior direction. Offline fibre tracking was performed independently by two observers (F.Z., M.P.), blinded to subject symptomatology and to prolapse status, using DTI software (DTITool, Biomedical Image Analysis group, Department of Biomedical Engineering, Eindhoven University of Technology, Eindhoven, The Netherlands [http:// bmia.bmt.tue.nl/software/dtitool]). Observer 1 (F.Z.), a 4thyear resident in radiology with additional 3-year experience as a teaching assistant at the Department of Anatomy and Embryology, Academic Medical Centre, University of Amsterdam, Amsterdam) and observer 2 (M.P., a medical doctor and 3rd-year PhD student) were experienced with the complex pelvic floor anatomy and subsequent 3D DTI tractography [8].

In the acquired FA maps and/or T1-weighted sequence, multiplanar, both manually drawn and positioned userdefined regions-of-interest (ROIs) were used to construct 3D fibre tracts of each of the predefined anatomical structures in the pelvic floor and wall (levator ani muscle \{i.e. pubovisceral, puborectal and iliococcygeus muscle [17]\}, anal and urethral sphincter complex, perineal body and internal obturator muscle) and the pelvic floor superficial layer (superficial transverse perineal, bulbospongiosus muscle [also called bulbocavernosus muscle] and ischiocavernosus muscles). As the pubovisceral muscle consists of different subdivisions (with insertions at the level of the vagina [pubovaginal muscle], perineal body [puboperineal muscle] and the anal sphincter complex [puboanal muscle], respectively [17]), observers were instructed to check whether these subdivisions could be separately visualised. Applied tracking parameters were standardised: minimal/ maximal fibre length $10-100 \mathrm{~mm}$; minimum/maximum FA thresholds $0.10-0.50$; angle threshold $<10-15^{\circ}$; integration step length 0.1 voxel. In the case of a paired anatomical structure, the left and right muscles were tracked separately. The two symmetrical parts of both the bulbospongiousus and the puboperineal muscle were considered as an unpaired structure for analytical purposes as these structures were difficult to isolate as a paired structure.

\section{Qualitative data analysis}

The resultant 3D representation of each isolated anatomical structure was independently rated by both observers using a four-point scale (good $=$ high-quality representation of the expected anatomical appearance within expected boundaries; sufficient $=$ adequate representation of the expected anatomical appearance, based on fibre orientation, shape and location, but presence of focal tracking distortions and non-tracking; insufficient $=$ non-satisfactory visualisation with presence of only a few fibre tracks and/or deviant fibre orientation, not found $=$ not present). Subsequently, rates were categorised into satisfactory (i.e. good and sufficient) and non-satisfactory (insufficient and not found). By using a consensus-based method, individual 3D fibre trajectories were qualitatively evaluated and ROIs were adjusted if necessary, resulting in a consensus-based dataset.

Towards a better understanding of qualitative and quantitative fibre tractography outcomes, one observer bilaterally evaluated the pubovisceral muscle on multiplanar 2D TSE $\mathrm{T} 2$-weighted images using a grading system that was previously reported to assess (birth-associated) muscle abnormalities [18]. A four-point Likert scale was used to qualitatively assess the pubovisceral musculature $(0=$ no visible defects; $1=$ less than half of muscle bulk lost; $2=$ more than half of muscle bulk lost; 3 = complete muscle bulk lost) [18].

\section{DTI parameters}

For quantitative analysis, mean values $( \pm \mathrm{SD})$ of the three eigen-values $(\lambda 1, \lambda 2$ and $\lambda 3)$ were calculated for multiple points per fibre along the resultant fibre tracts. Mean diffusivity (MD) and fractional anisotropy (FA) were defined as ( $D=$ diffusion tensor):

$\mathrm{MD}=\frac{1}{3} \operatorname{Trace}(\mathbf{D})=\left(\lambda_{1}+\lambda_{2}+\lambda_{3}\right) / 3=\langle\lambda\rangle$

$\mathrm{FA}=\sqrt{\frac{3}{2}} \frac{\sqrt{\left(\lambda_{1}-\langle\lambda\rangle\right)^{2}+\left(\lambda_{2}-\langle\lambda\rangle\right)^{2}+\left(\lambda_{3}-\langle\lambda\rangle\right)^{2}}}{\sqrt{\left(\lambda_{1}^{2}+\lambda_{2}^{2}+\lambda_{3}^{2}\right)}}$

Statistical analysis

Inter-observer agreement analysis was performed for each isolated anatomical structure. For the overall qualitative data 
(i.e. four-point scale quality assessment of 3D representation), inter-observer agreement was determined using quadratic weighted kappa statistics. Kappa statistics with 95\% confidence intervals were calculated. For qualitative subanalyses, prevalence- and bias-adjusted kappa (K) statistics (PABAK) were used.

DTI parameters (i.e. mean MD and FA values) were recorded and compared amongst the three groups if an isolated muscle was rated satisfactory (i.e. good or sufficient) in at least seven subjects per group. This cut-off was chosen to avoid statistically significant differences between groups based on substantial differences in group size. Permuscle means for MD and FA values were compared among the three groups using a one-way analysis of variance (ANOVA). In the event of a significant difference $(P<0.05$ ) a post-hoc Student's $t$-test with Bonferroni correction $(P<$ 0.016) was performed to study the significance among the sets of two groups. Inter-rater agreement for the per-muscle quantitative data between the two independent observers was assessed using intra-class correlation analysis (ICC).

\section{Results}

From January 2010 to December 2010, 30 women were included in this study. One subject was not willing to complete the MRI examination after a technical interruption of the acquisition procedure. In one subject image quality was poor and therefore fibre tractography was impossible, leaving 28 subjects for analyses. Baseline characteristics of the cohorts are shown in Table 1.

Qualitative analysis and inter-rater agreement

DTI with fibre-tractography was feasible in the 28 datasets, consequently resulting in 28 unpaired and 56 paired anatomical structures. Fibre-tractography resulted in a satisfactory anatomical representation of the pubovisceral muscle in $34 \%$ $(19 / 56)$; puborectal muscle in 13\% (7/56); superficial transverse perineal muscle in $27 \%$ (15/56); ischiocavernosus muscle in 54\% (30/56); bulbospongiosus muscle in 43\% (12/28) and urethral sphincter complex in $29 \%$ (8/28) of the datasets, respectively. No perceptible differences in tractability or nontractability were found in per-group distributions. The iliococcygeus muscle was rated non-satisfactory in all datasets (56/ 56). The following anatomical structures were identified in most of the DTI datasets: perineal body $100 \%(28 / 28)$, anal sphincter complex 93\% (26/28) and internal obturator muscle $100 \%$ (56/56) (Fig. 1). Despite the overall non-satisfactory visualisation of the global appearance of the pubovisceral muscle in the data sets (37/56), analyses of its subdivisions resulted in a satisfactory visualisation of the puboperineal muscle in 23 of the 28 subjects (Fig. 2). Both the pubovaginal and puboanal subdivision could not or only insufficiently be tracked.

Substantial overall inter-rater agreement was found for the independent qualitative scores. The overall weighted kappa for all muscle assessments was 0.71 (95\% CI 0.63 0.78). Qualitative inter-observer agreement for the anatomical structures which met the criteria for quantification was also substantial (PABAK $=0.76)$.

DTI parameters and inter-rater agreement

No statistical difference was detected in the mean values of MD between asymptomatic nulliparous women, age-matched controls and the prolapse group for the perineal body, anal sphincter complex, internal obturator muscle and the puboperineal muscle (Table 2). Mean MD values between groups ranged from $1.35 \pm 0.09 \times 10^{-3} \mathrm{~mm}^{2} / \mathrm{s}$ to $1.52 \pm 0.11 \times 10^{-3}$ $\mathrm{mm}^{2} / \mathrm{s}$ and FA values between $0.22 \pm 0.03$ to $0.27 \pm 0.04$. Analysis of variance demonstrated no significant differences in mean diffusivity between the three groups for the perineal body, anal sphincter complex and the puboperineal muscle
Table 1 Baseline characteristics of the study groups

Characteristics are expressed as median (range)

$B M I$ body mass index, $P O P-Q$ pelvic organ prolapse quantification, $P V M$ pubovisceral muscle

\begin{tabular}{llll}
\hline & Nulliparous group & Age-matched controls & Prolapse group \\
\hline Subjects per study group & 9 & 9 & 10 \\
Age & $28.8(20.8-32.3)$ & $52.6(43.7-65.3)$ & $59.4(45.0-69.9)$ \\
BMI & $21.5(20.5-26.3)$ & $22.2(19.9-31.4)$ & $24.3(22.2-35.1)$ \\
Parity & $0(0-0)$ & $2(0-3)$ & $2(1-3)$ \\
POP-Q stage & & & \\
Anterior compartment & $0(0-0)$ & $0(0-1)$ & $2(0-3)$ \\
Middle compartment & $0(0-0)$ & $0(0-1)$ & $1(0-2)$ \\
Posterior compartment & $0(0-0)$ & $1(0-1)$ & $1(0-2)$ \\
PVM injury & & $0(0-2)$ & $0(0-2)$ \\
Right & $0(0-0)$ & $0(0-1)$ & $1(0-2)$ \\
Left & $0(0-0)$ & &
\end{tabular}



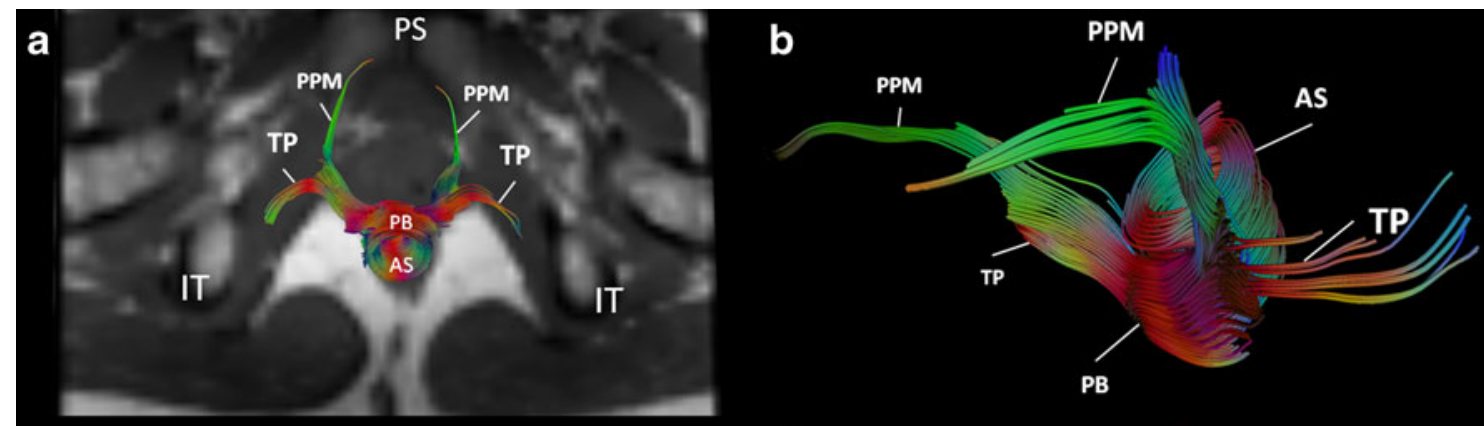

c

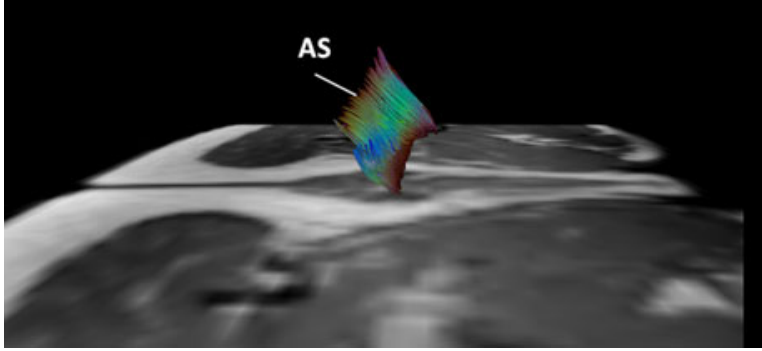

d

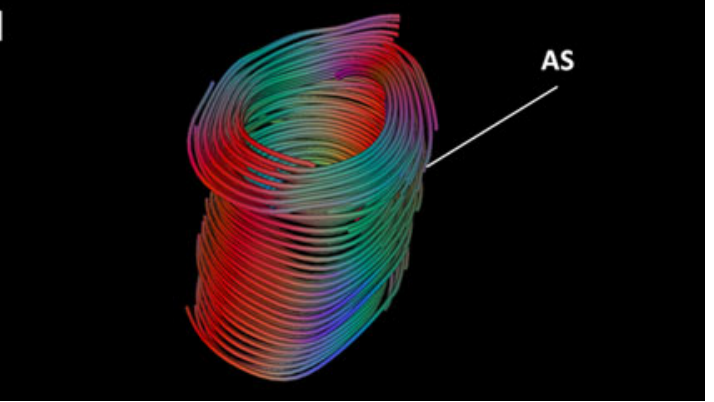

Fig. 1 a Axial T2-weighted image of the pelvic area (ischial tuberosity $[I T]$, pubic symphysis $[P S]$ ) of a 31-year-old nulliparous woman, with projected 3D fibre tractography. Fibre tracts represent the anal sphincter complex $(A S)$, perineal body $(P B)$, transverse perineal muscle $(T P)$ and puboperineal muscle $(P P M)$. Vector directions are colour coded: red indicating the left-right axis, blue the superior-inferior axis and green the antero-posterior axis. b An area of compact, horizontally

(Table 2). Mean FA value for the left obturator muscle was significantly lower in asymptomatic nulliparous women than in the prolapse group (mean FA $0.22 \pm 0.03$ and $0.27 \pm 0.05[P$ $<0.05]$, respectively), which consequently resulted in a significant difference between these groups for the combined obturator DTI measures (Table 2).

For the perineal body, anal sphincter complex, internal obturator muscle and the puboperineal muscle, the interrater agreement and $95 \%$ confidence interval $(\mathrm{CI})$ for mean diffusivity and FA are summarised in Table 3 . The intraclass correlation coefficient ranged between 0.60 and 0.91 , which was interpreted as a substantial inter-rater agreement. Highest correlation between the two observers was found for the perineal body.

The pubovisceral muscle status was normal for asymptomatic nulliparous women. Minor muscle injury (accumulated bilateral score 1-3, [18]) was observed for the age-matched control group. Both minor and major muscle injuries (accumulated bilateral score 4-6, [18]) were observed in the prolapse group. Median unilateral scores are expressed in Table 1.

\section{Discussion}

DTI permits 3D visualisation and quantification of part of the pelvic floor anatomy, with reliable high-quality tractability of the anal sphincter complex, perineal body, the puboperineal orientated fibre tracts $(\mathrm{red})$ is demonstrated at the level of the perineal body $(P B)$, when fibre tracts are viewed from left anterolaterally. $\mathbf{c}$ In the same patient, the vector direction on the level of the anal sphincter complex $(A S)$ reflects a predominating left-right (red) orientation at the anterior and posterior borders and combined anterior-posterior (green) and superior-inferior (blue) orientation at the lateral border, indicating circular orientation from right lateral (c) and left anterolateral views (d)

muscle - a subdivision of the pubovisceral muscle - and the internal obturator muscle. Basic DTI parameters could be determined with substantial inter-observer agreement. No apparent statistically significant differences in mean diffusivity (MD) and fractional anisotropy (FA) were demonstrated in the pelvic floor support between women with symptoms and stage 2 pelvic organ prolapse, women with symptoms and no clinically relevant prolapse and asymptomatic nulliparous controls.

DTI characterises and is able to visualise the local (micro)structural organisation within anisotropic tissues, such as brain white matter [19] and striated skeletal muscle [20]. The technical feasibility of 3.0-Tesla DTI with fibre tractography as a method of visualising the normal female pelvic anatomy was recently reported [8]. In that study, early estimates of the range of mean $\mathrm{MD}$ and FA values $(1.30 \pm$ $0.08 \times 10^{-3} \mathrm{~mm}^{2} / \mathrm{s}$ to $1.73 \pm 0.12 \times 10^{-3} \mathrm{~mm}^{2} / \mathrm{s}$ and $0.23 \pm 0.02$ to $0.30 \pm 0.04$, respectively) of pelvic floor structures in five healthy nulliparous women were provided. Current derived DTI parameters are concordant with these results.

The association between levator ani muscle impairment and the presence of pelvic floor prolapse has been recognised [3]. As DTI has been reported to be valuable in the assessment of muscle fatigue and defects [9], there was particular interest in the tractability of the pubovisceral muscle and its subdivisions (i.e. pubovaginal, puboperineal and puboanal muscle [17]), puborectal and iliococcygeus muscle in the present three study groups. It is known that the 

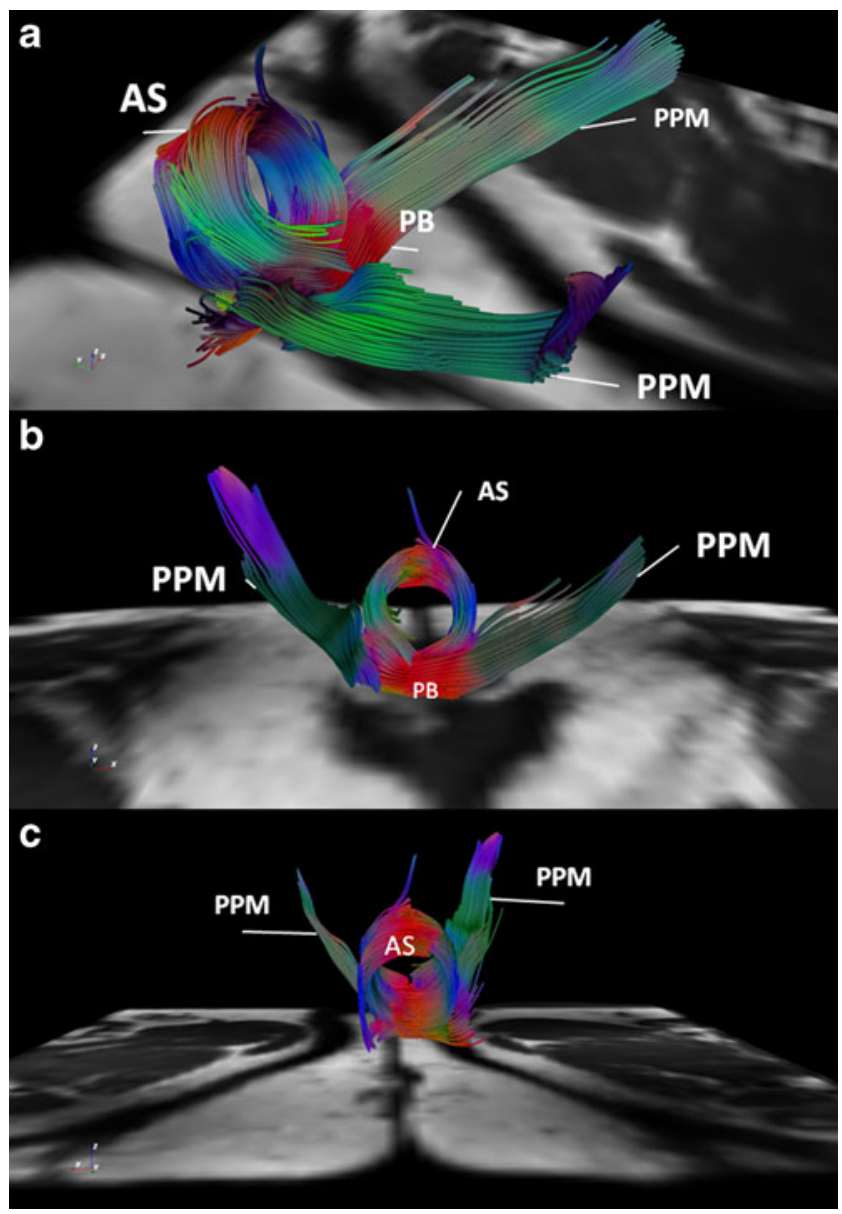

Fig. 2 a Tractography of the pelvic floor in a 70-year-old woman with pelvic organ prolapse, representing the anal sphincter complex $(A S)$, perineal body $(P B)$ and puboperineal muscle $(P P M)$ with an right anterolateral view, (b) anterior and (c) posterior view. Vector directions are colour coded: red $=$ left-right axis; blue = superior-inferior axis; green $=$ anterior-posterior axis. Fibre tracts originating from the pubic bone with posterior-inferior orientation of the vector as representation of the PPM reflected an overall blue and green colour-coded fibre tract. Both fibre tracts bend medially to insert into the PB

iliococcygeus muscle, the most posterior part of the levator ani muscle, is often poorly developed and consequently difficulties in fibre tracking were expected and were confirmed during analyses.

Based on the initial hypothesis, potential alterations in water diffusivity as measured in the levator ani musculature were expected to be found in the prolapse group and to a lesser extent in the age-matched group for the pubovisceral muscle. The pubovisceral muscle could be reliably tracked in a third of the women and the non-tractability of the pubovisceral musculature was proportionally distributed over the three study groups. In an earlier published pilot study in normal volunteers the pubovisceral muscle anatomy was globally demonstrated, although the highest variability in mean MD values was found for this muscle $\left(1.49 \pm 0.47 \times 10^{-3} \mathrm{~mm}^{2} / \mathrm{s}\right)$ and was attributed to its multifaceted organisation [8]. The relatively complex architecture of the pubovisceral muscle together with the relatively large voxel size and subsequent partial volume effects with potentially bending of effective diffusion vectors, made fibre tracking of the overall pubovisceral muscle in this study rather challenging with the currently applied MRI techniques. Despite these restrictions, assessment of the pubovisceral muscle resulted in a reliable 3D representation matching the global appearance of the puboperineal muscle anatomy, which appeared as robust fibre trajectories originating from the pubic bone and inserting into the perineal body. However, no significant differences were found in DTI measures among the groups.

Significantly higher mean FA values in the left internal obturator muscle were found for women in the prolapse group compared with nulliparous women. The principal function of the internal obturator muscle is to abduct the flexed thigh and although it forms the most important element of the pelvic wall, it is not regarded as a pelvic floor constituent. Yet, whereas the levator ani musculature anatomically originates partially from the tendinous ridge of the internal obturator muscle fascia, one might hypothesise a possible relation between levator ani muscular defects and subsequent changes in the internal obturator muscle on a microstructural level. In our study, most levator ani muscle defects [18] in the prolapse group were observed unilaterally on the left side.

There are considerable differences in size between the four isolated anatomical structures, as quantified and compared, in which the internal obturator muscle comprises a comparatively large muscular structure. Consequently, the mean number of fibres per isolated anatomical structure differs substantially among the anal sphincter complex, perineal body, the puboperineal muscle and the internal obturator muscle [8]. Whereas for each structure the DTI parameters are being calculated at multiple points per fibre along the fibre tracts, it seems reasonable to assume that subtle differences in architectural organisation are easier to detect in structures with a large number of fibre tracts. Detecting differences in DTI parameters in the closely aligned pelvic floor constituents is consequently challenged by both the relatively small number of fibre tracts and partial voluming effects that are more prominent in the smaller structures. We foresee that technical developments in DTI may overcome these limitations to at least a considerable extent in the near future.

The perineal body, located between the vagina and the anal sphincter complex, serves as an anchor point for the pelvic floor. Recently, the anatomy of the perineal body has been studied extensively by the Pelvic Floor Research Group with the use of thin-slice 3.0-T MRI, in an attempt to enhance our understanding of this centrally located anatomical structure [6]. In that study, 3D models were created based on conventional MRI acquisition methods in women without pelvic floor dysfunction, which allowed the 
Table 2 Mean diffusion tensor imaging (DTI) values \pm SD were compared among the nulliparous, control and prolapse groups. DTI measures for the internal obturator muscle were combined

$F A$ fractional anisotropy, $M D$ mean diffusivity

${ }^{\mathrm{a}}$ Units are: $\times 10^{-3} \mathrm{~mm}^{2} / \mathrm{s}$

${ }^{\mathrm{b}}$ Statistical differences among groups using one-way ANOVA test

${ }^{c}$ DTI measures of the left and right sides were combined

\begin{tabular}{llllllll}
\hline & $\lambda 1^{\mathrm{a}}$ & $\lambda 2^{\mathrm{a}}$ & $\lambda 3^{\mathrm{a}}$ & FA & $P$ value $^{\mathrm{b}}$ & $\mathrm{MD}^{\mathrm{a}}$ & $P$ value $^{\mathrm{b}}$ \\
\hline Perineal body & & & & & & & \\
Nullipara & $1.79 \pm 0.18$ & $1.35 \pm 0.16$ & $1.00 \pm 0.14$ & $0.25 \pm 0.06$ & 0.203 & $1.39 \pm 0.11$ & 0.877 \\
Controls & $1.67 \pm 0.18$ & $1.29 \pm 0.16$ & $1.03 \pm 0.16$ & $0.24 \pm 0.05$ & & $1.36 \pm 0.14$ & \\
Prolapse & $1.90 \pm 0.44$ & $1.50 \pm 0.38$ & $1.18 \pm 0.26$ & $0.24 \pm 0.06$ & & $1.51 \pm 0.36$ & \\
Anal sphincter & & & & & & & \\
Nullipara & $1.76 \pm 0.13$ & $1.37 \pm 0.11$ & $1.08 \pm 0.13$ & $0.25 \pm 0.04$ & 0.881 & $1.38 \pm 0.10$ & 0.340 \\
Controls & $1.76 \pm 0.30$ & $1.40 \pm 0.30$ & $1.09 \pm 0.22$ & $0.25 \pm 0.04$ & & $1.42 \pm 0.28$ & \\
Prolapse & $1.70 \pm 0.18$ & $1.36 \pm 0.11$ & $1.09 \pm 0.12$ & $0.22 \pm 0.03$ & & $1.39 \pm 0.12$ & \\
Internal obturator muscle & & & & & & & \\
Nullipara & & & & & & & \\
Controls & $1.80 \pm 0.16$ & $1.38 \pm 0.13$ & $1.12 \pm 0.10$ & $0.23 \pm 0.03$ & 0.013 & $1.43 \pm 0.13$ & 0.870 \\
Prolapse & $1.84 \pm 0.18$ & $1.39 \pm 0.17$ & $1.09 \pm 0.16$ & $0.25 \pm 0.04$ & & $1.44 \pm 0.18$ & \\
Puboperineal muscle & $1.91 \pm 0.27$ & $1.42 \pm 0.17$ & $1.08 \pm 0.15$ & $0.27 \pm 0.05$ & & $1.46 \pm 0.15$ & \\
Nullipara & & & & & & & \\
Controls & $1.71 \pm 0.10$ & $1.31 \pm 0.10$ & $1.00 \pm 0.09$ & $0.26 \pm 0.04$ & 0.127 & $1.35 \pm 0.09$ & 0.319 \\
Prolapse & $1.74 \pm 0.11$ & $1.37 \pm 0.15$ & $1.07 \pm 0.17$ & $0.27 \pm 0.04$ & & $1.39 \pm 0.14$ & \\
\hline & $1.81 \pm 0.27$ & $1.41 \pm 0.22$ & $1.14 \pm 0.19$ & $0.23 \pm 0.05$ & & $1.47 \pm 0.21$ & \\
\hline
\end{tabular}

complex anatomy to be organised into a superficial, mid and deep part [6]. With the use of a 5-mm slice thickness we were able to observe the large number of fibre tracts transversing and bending in this complex area. The perineal body anatomy or central perineal tendon [21] could be tracked in all subjects, demonstrating an area of compact horizontally orientated fibre trajectories located caudally in the midline between the urogenital area and anal sphincter complex. Given the increasing interest in the perineal body, detailed demonstration of this anchor point at DTI could be a potential advantage of this technique.

Because of the uniform left-right orientation of the mean diffusion tensor in this area, the perineal body could easily be

Table 3 Inter-rater agreement for mean diffusivity $(M D)$ and fractional anisotropy $(F A)$. Intra-class correlation coefficient $(I C C)$ and $95 \%$ confidence interval $(95 \% C I)$ are shown

\begin{tabular}{lcc}
\hline & ICC & $95 \% \mathrm{CI}$ \\
\hline Perineal body & & \\
FA & 0.82 & $0.65-0.91$ \\
MD & 0.91 & $0.81-0.96$ \\
Anal sphincter & & \\
FA & 0.76 & $0.55-0.88$ \\
MD & 0.60 & $0.30-0.79$ \\
Internal obturator muscle (R) & & \\
FA & 0.76 & $0.55-0.88$ \\
MD & 0.69 & $0.43-0.84$ \\
Internal obturator muscle (L) & & \\
FA & 0.70 & $0.44-0.85$ \\
MD & 0.74 & $0.52-0.87$ \\
Puboperineal muscle & & \\
FA & 0.71 & $0.44-0.86$ \\
MD & 0.66 & $0.37-0.84$ \\
\hline
\end{tabular}

identified on coloured FA maps in all subjects and therefore fibre tracts were obtained with high inter-rater agreement (ICC $>0.80$ for mean FA and MD values). This differed from the findings in the overall pubovisceral muscle, where fibre tract detection was much less apparent owing to its complex anatomy with closely aligned muscles, each with their own distinctive fibre direction. By assessing the different pubovisceral components during the analysis, we were able to overcome multidirectional tracking difficulties to a certain extent.

The DTI fibre tracking process is reported to be highly user-dependent, whereas the placement of the seeding ROI and the tracking criteria are determined by the individual observer [22]. By the use of predefined tracking parameters, which were applied by both observers, the fibre tract stopping criteria were standardised. Also, by the application of consensus-based reading, an attempt was made to reduce the user dependence of this method. For the anal sphincter complex, perineal body, puboperineal muscle and the internal obturator muscle, high intra-class coefficients were found, which can be interpreted as a substantial interobserver reliability of DTI measures.

This study has recognised limitations. At present, we were not able to compare DTI measures of all clinically relevant anatomical structures of the pelvic floor support among the three groups, owing to the limited fibre tractability of some anatomical structures. It is possible that injury of the pelvic floor musculature is more subtle and that DTI is not yet able to quantify this as effectively as in leg muscles [9]. Technical improvements may overcome these limitations. The current study was performed using a 3.0-Tesla system, 16-channel surface coil and optimised imaging parameters, but still relatively large voxel dimensions were acquired $(5 \times 2 \times 2=$ $20 \mathrm{~mm}^{3}$ ) compared with the commonly small diameter pelvic muscles. By increasing the number of acquisitions for the 
different encoding directions, an improvement of the signalto-noise ratio (SNR) and fibre-tracking precision can be obtained [23]. However, this will result in prolonged SE-EPI acquisition time (>10 min), which is associated with a higher likelihood of motion artefacts. Yet, spatial resolution can be optimised using dedicated coils, permitting the visualisation of smaller anatomical structures and to minimise spatial volume effects. The use of an endoanal coil will ultimately result in improved visualisation of the perineal body anatomy and anal sphincter complex, but it will distort the local anatomy while the small effective volume will preclude evaluation of the complete pelvic floor. The latter can be overcome by using a combined pelvic and endoanal coil. Endosonography is widely used in clinical practice and shown to be as accurate as MRI for evaluating anal sphincter lesions [4]. However, this does not allow simultaneous evaluation of the complete pelvic floor support as in MRI.

Based on the published feasibility study [8], we anticipated the detection of quantitative (micro)structural differences between the symptomatic and asymptomatic pelvic support even in a small sample size. Yet, our current findings may result from substantial per-subject variation in tissue characteristics.

In conclusion, DTI with fibre tractography allows for in vivo 3D visualisation of part of the pelvic floor support, with reliable visualisation of the anal sphincter complex, perineal body, puboperineal muscle and the internal obturator muscle in women with pelvic organ prolapse (POP) as well as in symptomatic age-matched controls and asymptomatic nulliparous women. No significant differences were found in extrapolated per-muscle DTI parameters of the pelvic support amongst the groups. Mean MD and FA values were assessed with substantial consistency by the different observers and overall SDs for the parameters were small. Further technical developments can be expected to lead to increased visualisation of those muscular structures that are not or only partly visualised in this series.

Open Access This article is distributed under the terms of the Creative Commons Attribution Noncommercial License which permits any noncommercial use, distribution, and reproduction in any medium, provided the original author(s) and the source are credited.

\section{References}

1. DeLancey JO, Kane Low L, Miller JM, Patel DA, Tumbarello JA (2008) Graphic integration of causal factors of pelvic floor disorders: an integrated life span model. Am J Obstet Gynecol 199:610.e1-610.e5

2. Lukacz ES, Lawrence JM, Contreras R, Nager CW, Luber KM (2006) Parity, mode of delivery, and pelvic floor disorders. Obstet Gynecol 107:1253-1260
3. DeLancey JO, Morgan DM, Fenner DE et al (2007) Comparison of levator ani muscle defects and function in women with and without pelvic organ prolapse. Obstet Gynecol 109:295-302

4. Dobben AC, Terra MP, Slors JF et al (2007) External anal sphincter defects in patients with fecal incontinence: comparison of endoanal MR imaging and endoanal US. Radiology 242:463-471

5. El Sayed RF, El Mashed S, Farag A, Morsy MM, Abdel Azim MS (2008) Pelvic floor dysfunction: assessment with combined analysis of static and dynamic MR imaging findings. Radiology 248:518-530

6. Larson KA, Yousuf A, Lewicky-Gaupp C, Fenner DE, DeLancey JO (2010) Perineal body anatomy in living women: 3-dimensional analysis using thin-slice magnetic resonance imaging. Am J Obstet Gynecol 203:494.e15-494.e21

7. Boyadzhyan L, Raman SS, Raz S (2008) Role of static and dynamic MR imaging in surgical pelvic floor dysfunction. Radiographics 28:949-967

8. Zijta FM, Froeling M, van der Paardt MP et al (2011) Feasibility of diffusion tensor imaging (DTI) with fibre tractography of the normal female pelvic floor. Eur Radiol 21:1243-1249

9. Zaraiskaya T, Kumbhare D, Noseworthy MD (2006) Diffusion tensor imaging in evaluation of human skeletal muscle injury. J Magn Reson Imaging 24:402-408

10. Kan JH, Heemskerk AM, Ding Z et al (2009) DTI-based muscle fiber tracking of the quadriceps mechanism in lateral patellar dislocation. J Magn Reson Imaging 29:663-670

11. Bump RC, Mattiasson A, Bø K et al (1996) The standardization of terminology of female pelvic organ prolapse and pelvic floor dysfunction. Am J Obstet Gynecol 175:10-17

12. Uebersax JS, Wyman JF, Shumaker SA, McClish DK, Fantl JA (1995) Short forms to assess life quality and symptom distress for urinary incontinence in women: the incontinence impact questionnaire and the urogenital distress inventory. Neurourol Urodyn 14:131-139

13. van Brummen HJ, Bruinse HW, van de Pol G, Heintz AP, van der Vaart CH (2006) Defecatory symptoms during and after the first pregnancy: prevalences and associated factors. Int Urogynecol J Pelvic Floor Dysfunct 17:224-230

14. Froeling M, Nederveen AJ, Heijtel DF et al (2012) Diffusiontensor MRI reveals the complex muscle architecture of the human forearm. J Magn Reson Imaging 36:237-248

15. Aja-Fernandez S, Niethammer M, Kubicki M, Shenton ME, Westin CF (2008) Restoration of DWI data using a Rician LMMSE estimator. IEEE Trans Med Imaging 27:1389-1403

16. Leemans A, Jones DK (2009) The B-matrix must be rotated when correcting for subject motion in DTI data. Magn Reson Med 61:1336-1349

17. Umek WH, DeLancey JO (2008) MRI of the levator ani muscle. In: Stoker J, Taylor S, DeLancey JO (eds) Imaging pelvic floor disorders. Springer, New York, pp 89-111

18. DeLancey JO, Kearney R, Chou Q, Speights S, Binno S (2003) The appearance of levator ani muscle abnormalities in magnetic resonance images after vaginal delivery. Obstet Gynecol 101:46-53

19. Nucifora PG, Verma R, Lee SK, Melhem ER (2007) Diffusiontensor MR imaging and tractography: exploring brain microstructure and connectivity. Radiology 245:367-84

20. Froeling M, Oudeman J, van den Berg S et al (2010) Reproducibility of diffusion tensor imaging in human forearm muscles at 3.0 $\mathrm{T}$ in a clinical setting. Magn Reson Med 64:1182-1190

21. Stoker J, Wallner C (2008) The anatomy of the pelvic floor and sphincters. In: Stoker J, Taylor S, De Lancey JOL (eds) Imaging pelvic floor disorders. Springer, New York, pp 1-29

22. Brecheisen R, Platel B, Vilanova A, ter Haar RB (2009) Parameter sensitivity visualization for DTI fiber tracking. IEEE Trans Vis Comput Graph 15:1441-8

23. Khalil C, Budzik JF, Kermarrec E, Balbi V, Le Thuc V, Cotten A (2010) Tractography of peripheral nerves and skeletal muscles. Eur J Radiol 76:391-397 